\title{
Influencia de la formación universitaria en las actitudes emprendedoras*
}

\author{
The influence of higher education on entrepreneurial attitudes
}

\author{
Juan Luis Reyes-Cruz \\ Doctorando en Ciencias de la Administración, Universidad Autónoma del estado de Hidalgo, \\ Pachuca-México, jlreyes_13@hotmail.com \\ Magda Gabriela Sánchez-Trujillo \\ Doctora en Administración, Universidad Autónoma del estado de Hidalgo, \\ Pachuca-México, mgabyst@gmail.com \\ Raúl Mejía-Ramírez \\ Doctor en Ciencias de la Administración, Instituto Tecnológico Superior de la Costa Chica, \\ Ometepec-México, raul_mejia81@hotmail.com
}

\begin{abstract}
Cómo citar / How to cite
Reyes-Cruz, J. L., Sánchez-Trujillo, M. G. \& Mejía-Ramírez, R. (2019). Influencia de la formación universitaria en las actitudes emprendedoras. Revista CEA, 5(10), 117-133. https://doi.org/10.22430/24223182.1240

Recibido: 9 de abril de 2019

Aceptado: 27 de junio de 2019
\end{abstract}

\section{Resumen}

El objetivo de este trabajo es caracterizar la influencia de la formación universitaria en las actitudes emprendedoras, a partir de la adquisición de rasgos dinámicos de innovación, propensión al riesgo, personalidad, aspectos psicológicos y demográficos. Para ello, el proceso estuvo compuesto por 4 dimensiones con 75 ítems. La recolección de datos se realizó in situ a los estudiantes en cuatro universidades. La metodología empleada consiste en la técnica de redes neuronales artificiales, en la que se ajustó un modelo de tipo Perceptrón Multicapa (PM), mediante el software Economática. Los resultados muestran que el desarrollo del proceso emprendedor está determinado, principalmente, por la formación práctica de los estudiantes que les permite aplicar los conocimientos adquiridos en empresas y organismos de la región o bien en la incubación de negocios en la institución, así como obtener ingresos extras, vía participación en proyectos de investigación y que tengan disciplina de práctica deportiva.

Palabras clave: enseñanza universitaria, redes neuronales, estudiantes universitarios, emprendimiento, intención emprendedora, educación emprendedora.

*Este artículo se deriva del proyecto titulado «Influencia de la formación universitaria en las actitudes emprendedoras» y ha sido financiado con recursos propios. 


\begin{abstract}
The objective of this work is to characterize the influence of higher education on the entrepreneurial attitudes of undergraduate students by documenting their dynamic traits of innovation, risk propensity, and personality, as well as psychological and demographic aspects. For that purpose, the process was divided into 4 dimensions with 75 items. The data of students enrolled in four different universities were collected in situ. This study adopted the technique of artificial neural networks and an adjusted Multilayer Perceptron (MP) by means of the software Economática. The results show that the development of the entrepreneurial process is mainly determined by three aspects: (1) their hands-on training, which enables students to apply their knowledge at local companies and organizations or incubate businesses at their institution; (2) receiving additional income by participating in research projects; and (3) playing sports.
\end{abstract}

Keywords: University teaching, neural network, university students, entrepreneurship, entrepreneurial intention, entrepreneurial education.

\title{
1. INTRODUCCIÓN
}

El ambiente competitivo de los países exige una mejor preparación de los estudiantes en su formación universitaria, que les permita al egresar contar con opciones de desempeño profesional, ya sea desempeñarse en una empresa o bien generar su propio negocio y auto emplearse. La nueva perspectiva económica de América Latina, según la Organización para la Cooperación y el Desarrollo Económico (OCDE), está centrada en un sustancial bono demográfico (un fenómeno que se da cuando el volumen de la población en edad de trabajar, que se ubica entre 14 y 59 años, supera a la población dependiente -niños y ancianos-), la formación basada en competencias y el emprendimiento que busca un desarrollo orientado en la inclusión y sustentabilidad en la región.

En este sentido, de aprovecharse adecuadamente el bono demográfico de América Latina, que es el más grande del mundo, podría representar una ventaja competitiva para los países, ya que uno de cada cuatro latinoamericanos tiene entre 15 y 29 años, lo que suma 163 millones de personas en edad productiva, además, dos de cada diez de estos jóvenes trabajan en el sector informal y otro $20 \%$ no trabaja, ni estudia, lo que significa que no están recibiendo instrucción o bien la recibieron y no la provechan en actividades productivas (Organización para la Cooperación y el Desarrollo Económicos - OCDE, Banco de Desarrollo de América Latina - CAF y Comisión Económica para América Latina y el Caribe - CEPAL, 2016). Este panorama no es alentador, si se suma el fenómeno de desaceleración económica que limita las oportunidades, tanto de empleo, así como las de emprendimiento.

El Banco Mundial (2016) resalta el impacto de la actividad emprendedora en el desarrollo económico en el mundo, de ahí que resulte interesante analizar la relación que guarda la formación recibida en las universidades con el papel de dichos emprendedores, para aprovechar el potencial de los jóvenes y utilizar su talento y energía, tanto en proyectos innovadores, como en las empresas existentes, ya que con ello se podría promover la generación de empleo, el crecimiento económico y la movilidad social en las regiones. Así, las Instituciones de Educación Superior (IES), entre ellas, la Universidad Nacional de México (UNAM) han declarado que forman, o al menos contribuyen a la formación de 
emprendedores en el desarrollo de sus características, con programas de emprendimiento, asignaturas, incubadoras de empresas y el perfil de sus académicos.

Por su parte, en México, tras identificar que se ocupaba el lugar 72 entre al menos 144 países (Lederman, Messina, Pienknagura y Rigolini, 2014), se implementaron políticas de fomento a los emprendedores en busca el impulsar la economía, fortalecer el mercado interno y la innovación, así mismo, el gobierno mexicano estimula la participación de las mujeres en el emprendimiento a través de la asistencia técnica, fomento a proyectos sociales, verdes y de alto impacto, que faciliten el incremento de la masa crítica de emprendedores.

Por lo anterior, el objetivo central del presente trabajo es caracterizar el proceso emprendedor mediante los rasgos de innovación, propensión al riesgo, personalidad, aspectos psicológicos y demográficos y su incidencia en la formación académica. Para alcanzar dicho objetivo es necesario responder los siguientes interrogantes:

1. ¿La formación universitaria incide directamente con el proceso emprendedor?

2. ¿Los programas académicos de Administración de las Universidades Autónoma del Estado de Hidalgo, Autónoma Metropolitana, Nacional Autónoma de México y Autónoma de Tlaxcala, ¿han adquirido características emprendedoras en su formación curricular?

\section{MARCO TEÓRICO}

\section{Emprendedor y emprendimiento}

El estudio precursor sobre el emprendedor aparece por primera vez en el texto de Cantillon (1955), denominado Ensayo sobre la naturaleza del comercio en general, quien definió emprendedor, como la persona que organiza y asume el riesgo en un negocio esperando compensarlo con las utilidades. Posteriormente, Say (1821), introdujo el término entrepreneur, definiéndolo como la persona que toma riesgos, asume responsabilidad inmediata y conforma una actividad económica, ya sea sobre su propio capital o de un capital prestado.

Más adelante, Schumpeter (1934) afirma que cada emprendedor es único cuando lleva a cabo nuevas combinaciones de medios de producción y crean así nuevos productos, métodos de producción, mercados, fuentes de aprovisionamiento o modalidades de organización, y estas inducen a la destrucción creativa del antiguo orden, provocando cambios en el crecimiento económico.

Por su parte, Ross (1995) define a los emprendedores como productores que introducen por primera vez un bien o una nueva combinación de factores, mientras que Benavente (2004) precisa que el emprendedor es el dueño de empresas jóvenes entre 3 y 10 años, que tienen entre 15 y 300 trabajadores; más adelante, Buelna \& Ávila (2009) mencionan que los humanos en general son una especie emprendedora, porque en el transcurso de su existencia han generado patrones de conducta relacionados con la innovación.

Respecto al concepto emprendedor, se puede reconocer que su formación y desarrollo depende de distintos factores. Existen al menos dos corrientes sobre la formación y desarrollo de 
emprendedores: la primera, dice que el emprendedor posee ciertas características que lo hacen diferente de otras personas (Hornaday \& Aboud, 1971). La otra posición, plantea que el emprendedor se puede formar desde un punto de vista social (Gibb \& Ritchie, 1982), o bien, el emprendedor se forma y es producto de su experiencia adquirida y entrenamiento, medios por los cuales aprende a pensar empresarialmente. Para efectos de este trabajo, se entenderá como emprendedor a los individuos capaces de producir un bien o servicio con el fin de obtener un bien económico partiendo de la idea que el emprendedor se puede formar.

Respecto al emprendimiento existen diversos estudiosos que explican el término. Para Van Praag (1999) el riesgo define al emprendedor; por su parte, Benavente (2004) plantea que la propiedad de una empresa o negocio define al emprendedor. Existe otra postura que asocia el emprendimiento con la innovación (Cadavid, Díez-Echavarría \& Valencia-Arias, 2017). En este contexto, el emprendimiento es reconocido como línea de investigación independiente, y es cada vez más creciente y consistente el interés por su estudio científico (Mejía Ordoñez, Arias Marin \& Echeverry Sánchez, 2017).

Estudios contemporáneos manifiestan que a mayor nivel educativo alcanzado la persona presenta una mayor probabilidad de establecer negocios propios. En ese sentido, se encuentran los resultados arrojados por Giannetti \& Simonov, (2004), Glocker \& Steiner (2007), Leal y Cepeda (2015), Estrin, Mickiewicz, \& Stephan (2016), García, Martínez y Fernández (2007). También, se observan investigaciones que reflexionan lo opuesto, en donde a mayor nivel educativo se reduce la probabilidad de realizar emprendimientos (Santana Vega, González-Morales \& García, 2016).

Otro aspecto importante a considerar en el fenómeno del emprendimiento es la experiencia; las personas que crean negocios se caracterizan por poseer mayores años de experiencia laboral acumulada, ya que esta contribuye positivamente a la acción emprendedora, según los trabajos de Hernández-López, Moncada-Toro \& Henao-Colorado (2018).

Respecto a la causalidad entre el nivel personal y el emprendimiento, algunas investigaciones determinan la existencia de una relación en sentido positivo; en tanto que otras, una relación negativa. Entre los que determinan una causalidad en sentido positivo están los trabajos de Glocker \& Steiner (2007), Doh \& Zolnik (2011) y Capelleras Segura, Contín Pilart, Martín Sánchez \& Lazarra Kintana. En contraparte se encuentran aquellos estudios que especifican una relación inversa Giannetti \& Simonov (2004).

Una vez confirmada la creciente importancia del proceso emprendedor, resulta pertinente analizar si la formación universitaria, en el caso de México, fomenta, posibilita o influye en la creación de nuevas empresas.

\section{Características emprendedoras}

Para Fuentes-García \& Sánchez-Cañizares (2010), el emprendedor es determinante de la innovación y pauta del crecimiento económico. Los autores aluden a que se puede estudiar en tres dimensiones: su perfil demográfico, su perfil psicológico y su perfil sociológico. De acuerdo con estos autores, el perfil del emprendedor es mejor explicado por variables psicológico-sociales, de acuerdo con sus resultados (Espíritu \& Moreno, 2010). 
Entre los principales factores demográficos, se destacan la edad y el sexo, formación y experiencia (Fayolle, Vernier \& Djiane, 2004), consideran que los rasgos demográficos sobresalen por su importancia en la toma de decisiones estratégicas. En ese sentido, McClelland (1961), identifica tres principales rasgos: la motivación al logro, el grado de propensión al riesgo y el control interno.

El enfoque sociológico plantea, además, factores externos al emprendedor, tales como la influencia de amigos y familiares (capital social), así como la influencia de padres empresarios (Scott \& Twomey, 1988). En este sentido, en la tabla 1, se evidencia que el perfil emprendedor está determinado por antecedentes familiares, como el orden de nacimiento, estado civil, edad, o bien el nivel educacional de sus padres (Lazear, 2005).

Tabla 1. Características de emprendedores según autores Table 1. Entrepreneur traits

\begin{tabular}{ccc}
\hline $\begin{array}{c}\text { Psicológicas } \\
\text { (Mc Clelland, 1961) }\end{array}$ & $\begin{array}{c}\text { Demográficas } \\
\text { (Sudek, 2006) } \\
\text { Originalidad e innovación }\end{array}$ & $\begin{array}{c}\text { Sociológicas } \\
\text { (Lazear, 2005) (Ajzen, 1991) }\end{array}$ \\
\hline $\begin{array}{c}\text { Moderada aversión al riesgo de logro } \\
\text { Aceptación de sus } \\
\text { responsabilidades }\end{array}$ & Autoconfianza & Sexo \\
\hline $\begin{array}{c}\text { Conocimiento de los resultados de } \\
\text { sus actos }\end{array}$ & Optimismo & Formación \\
\hline Planificación & Creatividad & Experiencia \\
\hline
\end{tabular}

Fuente: elaboración propia a partir de McClelland (1961), Sudek (2006), Lazear (2005) y Ajzen (1991).

En esta investigación, se considera además del perfil sociológico y las competencias sociales, ya que muestran la forma en que el emprendedor toma iniciativas y se relaciona con los demás.

\section{Formación curricular}

Para determinar la influencia de la formación curricular en la adquisición de características emprendedoras es necesario definir el concepto de formación. De acuerdo con el Diccionario de la Real Academia de la Lengua Española (2019), 'formación' es "acción y efecto de formar», entendiendo el verbo formar en la concepción de «dar forma»; desde el punto de vista pedagógico y cultural, la formación se puede describir desde la concepción del hombre hasta su desarrollo, como un ser en evolución y constante transformación.

En estos aspectos, Quiceno (1996), lo describe como la evolución de la educación que va teniendo el hombre a lo largo de su vida; en lo cultural, son los valores, creencias, y modelos que tenga el hombre sobre sí mismo y que son transmitidos por la cultura dominante, en su función social de transmisión (Ferry, Wieder \& Jiménez Silva, 1991), de las generaciones adultas a las más jóvenes, ya sea por medio de la educación académica y profesional o la educación en el entorno social en el que se desenvuelve el individuo, de tal manera que la formación puede ser construida a través de la enseñanza de las disciplinas, saber y ciencias (Reboul, 1989). 
Por tanto, la formación es crucial en el desarrollo personal y profesional de cualquier persona, por el impacto que genera en distintos niveles y en diversas actividades, como el emprendimiento.

\section{METODOLOGÍA}

Se trata de un estudio cuantitativo, de alcance descriptivo y relacional. Para caracterizar el proceso de las características emprendedoras en los universitarios de la presente investigación, se plantea la hipótesis general: H1 La formación universitaria está relacionada con el comportamiento emprendedor en los alumnos de la Licenciatura en Administración de universidades públicas mexicanas.

\section{Población y Muestra}

Los criterios de inclusión son:

Universidades donde se impartiera la Licenciatura de Administración, en la zona centro de México, las cuales son: UNAM, UAM, UAEH, UAT.

Porcentaje de $60 \%$ mínimo de avance curricular (de sexto semestre en adelante).

Tener contacto y aprobación con los docentes que imparten materias en dichos semestres, para solicitar aprobación para aplicar instrumento a estudiantes.

Estudiantes que hubieran cursado al menos una asignatura relacionada o enfocada al emprendimiento (ver Tabla 2).

Tabla 2. Población universitaria

Table 2. Number of students

\begin{tabular}{cc}
\hline Universidades & $\begin{array}{c}\text { Matrícula en Lic. } \\
\text { Administración }\end{array}$ \\
\hline Universidad Autónoma del Estado de Hidalgo & 1.735 \\
\hline Universidad Nacional Autónoma de México & 9.075 \\
\hline Universidad Autónoma Metropolitana & 3.300 \\
\hline Universidad Autónoma de Tlaxcala & 765 \\
\hline Total & 14.875 \\
\hline
\end{tabular}

Dadas las condiciones de disponibilidad para responder el instrumento, la selección de la muestra fue por conveniencia, por lo que estuvo compuesta 297 alumnos de administración de las cuatro universidades públicas mexicanas. 


\section{Medición de variables}

\section{Características emprendedoras (variable dependiente)}

Se busca identificar el desarrollo de características emprendedoras en los universitarios que participaron como muestra, tomando de base características validadas, como son: innovación, propensión al riesgo, personalidad, aspectos psicológicos y demográficos. La propensión al riesgo es inherente al emprendedor para explorar oportunidades de alcanzar objetivos y asumir su responsabilidad implícita (Espíritu \& Moreno, 2010), para controlar su propio destino y los resultados de los objetivos impuestos, definiéndolo como control interno (Ayerbe \& Buenetxea, 2000).

\section{Formación Curricular (variable independiente)}

En esta variable se mide la influencia de los programas académicos, asignaturas y perfil del docente en la adquisición de características emprendedoras. A través del instrumento, se recaba información sobre la influencia de la formación curricular que adquieren los alumnos en las universidades de estudio.

\section{Instrumento}

Se utilizó un instrumento validado por Reyes-Cruz, Garzón-Castrillón \& Tapia-Sánchez, (2018), compuesto por cuatro dimensiones: características demográficas, aspectos sociales, características emprendedoras y formación universitaria, con 75 ítems con opciones de respuesta: nunca, rara vez, algunas veces, casi siempre y siempre. La recolección de datos se realizó in situ a los estudiantes en cada universidad, lo que facilitó la aplicación; el procesamiento de la información se realizó con ayuda del software Economática.

\section{Análisis estadístico}

La metodología empleada se basa en la técnica de redes neuronales artificiales.

Los modelos de regresión estudian la relación entre una serie de variables denominadas independientes $x$, y otras variables dependientes o respuesta, que se denotan como $y$. El objetivo es predecir los valores de $y$ en función de los valores de $x$. El modelo básico se expresa como:

$$
n=\sum_{i=0}^{N} \beta i X i(1)
$$

Donde $\mathrm{n}=h(\mu) \mathrm{n} E(y) n=n$

En estas expresiones $h(\mu)$ es la función que liga los componentes, $\beta$ son los coeficientes, $n$ es el número de variables independientes y ßo es la pendiente.

El modelo lineal se puede implementar como una red neuronal simple: tiene una unidad de sesgo, una unidad de input y una unidad de salida. El input se refiere a una variable $x$, mientras que el sesgo siempre es una constante igual a 1. El output sería: 


$$
y 2=y 1 w 21+1,0 w 20(2)
$$

Existen diferentes modelos neuronales artificiales, pero una característica común que tienen la mayoría de estas es que tienen el siguiente proceso de operación bajo la conceptualización de un sistema (Pérez \& Martín, 2003) (Ver Figura 1).

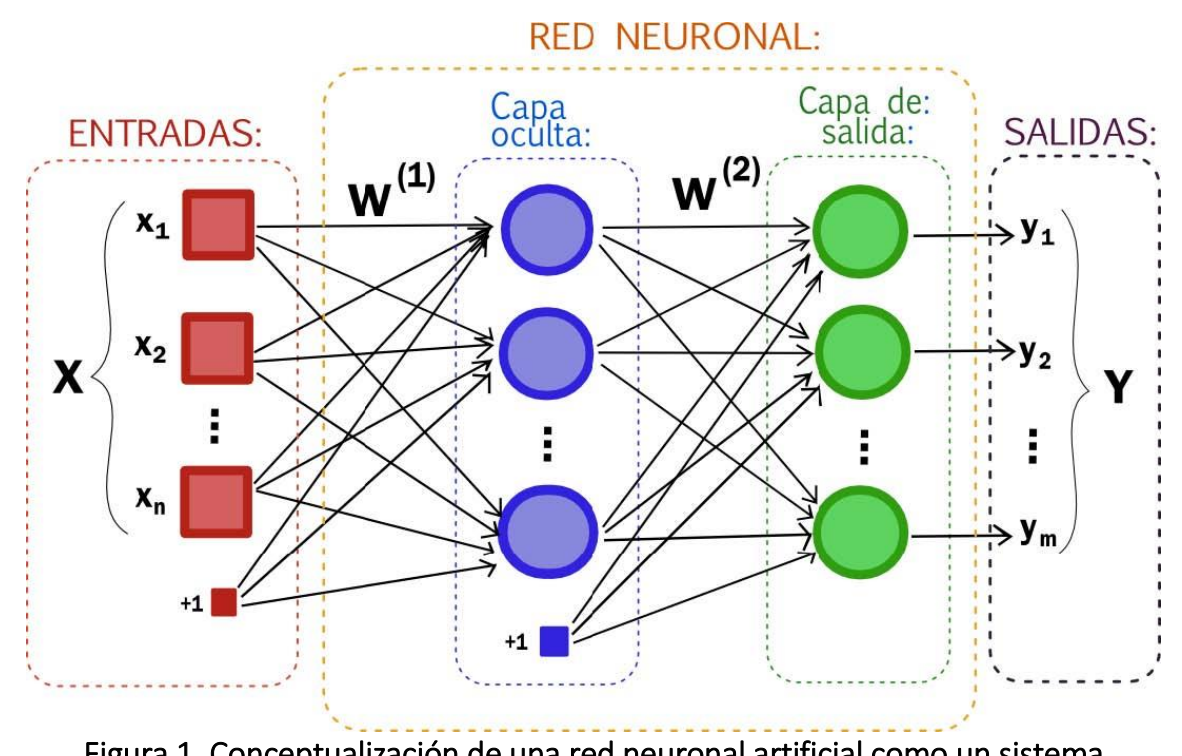

Figura 1. Conceptualización de una red neuronal artificial como un sistema

Figure 1. Conceptualization of an Artificial Neural Network as a System

Fuente: elaboración propia.

Al igual que el concepto de un sistema en que existe entrada, una unidad de proceso y una salida, una red neuronal funciona de la misma manera. De la Figura 1, se puede decir que una Red Neuronal Artificial (RNA), en cada elemento de proceso, tiene un conjunto de entradas y una sola salida por las que circulan las señales. Estas señales dependen del instante de tiempo considerado.

Estas variables, tanto las de entrada como las de salida, pueden ser discretas o continuas, dependiendo del modelo de neurona considerada y de la aplicación que se le vaya a dar. Cuando las salidas pueden tomar valores continuos, se suelen limitar a un intervalo definido, entre cero y uno.

Como se aprecia en la Figura 1, la entrada de la RNA, tiene una conexión de entrada que está asociada a un peso (w), que determina el efecto cuantitativo de unas unidades sobre otras y corresponde a las sinapsis del sistema. Estos pesos se suelen representar con una $W_{-} i^{\wedge}$, en que los dos subíndices que indican la neurona (i) que le corresponde y la entrada (j), a dicha neurona a la que están asociados, respectivamente. Por tanto, cada neurona tendrá tantos pesos como entradas.

Para que exista conexión entre la entrada, esta se determina aplicando una regla de propagación bajo una combinación lineal, entre las entradas y sus correspondientes pesos, como se indican en la ecuación (1). Donde (i) representa el elemento de proceso cuya entrada neta se calcula, (n) es el número de entradas de dicho elemento de proceso, las entradas se representan con una $(\mathrm{x})$ y los pesos con una (w) (Pérez \& Martín, 2003). 
Dentro del elemento de proceso o caja negra como lo indica la teoría de sistemas, García (2018) para cada elemento de proceso en un instante de tiempo determinado tiene asociado un valor de activación, $\theta \_i(t)$. Su nuevo valor de activación se determina aplicando una función (f), denominada función de activación. Para una neurona artificial esta función se considera determinista.

Existen diversos tipos de redes neuronales, sin embargo, no se pretende en este trabajo profundizar sobre las diferentes arquitecturas (construcción de las redes neuronales), ya que no es el propósito de esta investigación.

Para lograr el objetivo de esta investigación, se utilizan variables cuantitativas, tanto para las entradas como para las salidas, y las RNA reconocen patrones de aprendizaje de los diferentes grupos homogéneos formados con base en los resultados de la variable dependiente, que en este caso es binaria (1 para la persona emprendedora y 0 para la persona no emprendedora) y se determinan las variables de formación universitaria que intervienen en ello.

\section{RESULTADOS}

Como ya se mencionó, en la actualidad existen trabajos que abordan la importancia de la formación emprendedora o educación emprendedora como tema relevante, ya sea debido a su papel para generar cultura emprendedora o aquellos que miden la intención que tengan de emprender los estudiantes (Entrialgo e Iglesias, 2016).

Distintos trabajos plantean la formación empresarial como medio para que los estudiantes inicien su propio negocio, como alternativa para la incorporación exitosa en el mercado (Herman \& Stefanescu, 2017), (Hamzah, Yahya, Sarip \& Adnan, 2016). Para la contribución en la discusión del tema, posterior al cálculo de los datos, la Tabla 3 muestra el resumen del proceso de los 297 casos de la muestra, para clasificar emprendedor de no emprendedor, en relación con la formación universitaria, para ello se entrena a la red con una muestra denominada de entrenamiento conformada por 131 casos, una muestra de prueba con 50 y una de reserva con 478.

La muestra de entrenamiento comprende los registros de datos utilizados para entrenar la red neuronal, un cierto porcentaje de casos del conjunto de datos se asignan a la muestra de entrenamiento para poder obtener un modelo. La muestra de prueba es un conjunto independiente de registros de datos que se utiliza para realizar un seguimiento de los errores durante el entrenamiento, se recomienda crear una muestra de entrenamiento cuando la muestra de prueba es menor que esta, con el fin de hacer más eficiente la prueba.

La muestra reservada es otro conjunto independiente de registros de datos que se utiliza para evaluar la red neuronal final; el error de la muestra reservada ofrece una estimación clara de la capacidad de clasificación del modelo, ya que los casos reservados no se utilizan para crear el modelo. 
Tabla 3. Resumen del procesamiento de casos Table 3. Case processing summary

\begin{tabular}{cllc}
\hline & & N & Porcentaje \\
\hline Muestra & & 297 & \\
& Entrenamiento & 131 & $19.9 \%$ \\
Válidos & Prueba & 50 & $8.0 \%$ \\
& Reserva & 478 & $72.0 \%$ \\
Excluidos & 0 & $100.0 \%$ \\
Total & & 659 & \\
Fuente: elaboración propia a partir de la información procesada en Economática.
\end{tabular}

La información sobre la red neuronal resulta útil para garantizar que las especificaciones son correctas. En los resultados es posible observar:

1. El número de unidades en la capa de entrada es el número de covariables, más el número total de niveles de factor. Se crea una unidad independiente para cada categoría de desempeño y ninguna de las categorías se considera como una unidad «redundante», como es habitual en muchos procedimientos de creación de modelos.

2. De igual manera, se crea una unidad de resultado independiente para cada categoría de desempeño, para un total de dos unidades en la capa de resultados.

3. La selección de arquitectura automática ha elegido cinco unidades en la capa oculta.

4. El resto de la información de red se toma por defecto para el procedimiento (Ver anexo 1).

La Tabla 4 muestra información sobre los resultados de entrenar y aplicar la red final a la muestra reservada. En dicho cuadro se puede observar:

1. El error de entropía cruzada es la función de error que la red intenta minimizar durante el entrenamiento.

2. El porcentaje de pronósticos incorrectos se toma de la tabla de clasificación, y se discutirá más adelante en ese tema.

3. El algoritmo de estimación se ha detenido, ya que se ha alcanzado el número máximo de épocas. Lo ideal es que el entrenamiento se detenga, puesto que el error ha convergido. Esto plantea cuestiones sobre si se ha producido algún error durante el entrenamiento, y se debe tener en cuenta al realizar una inspección adicional de los resultados.

Tabla 4. Resumen del modelo

Table 4. Model summary

\begin{tabular}{|c|c|c|}
\hline \multirow[t]{4}{*}{ Entrenamiento } & Error de entropía cruzada & 155,260 \\
\hline & Porcentaje de pronósticos incorrectos & $23,2 \%$ \\
\hline & Regla de parada utilizada & $\begin{array}{l}1 \text { paso(s) consecutivo(s) sin } \\
\text { disminución del error }\end{array}$ \\
\hline & Tiempo de entrenamiento & 0:00:00,30 \\
\hline \multirow[t]{2}{*}{ Pruebas } & Error de entropía cruzada & 69,799 \\
\hline & Porcentaje de pronósticos incorrectos & $24,4 \%$ \\
\hline \multirow[t]{2}{*}{ Reserva } & Porcentaje de pronósticos incorrectos & $28,0 \%$ \\
\hline & Variable dependiente: Emprended & \\
\hline \multicolumn{3}{|c|}{ * Los cálculos de error se basan en la muestra de comprobación. } \\
\hline
\end{tabular}


Se observa consistencia en cada una de las pruebas al obtener más del $70 \%$ de clasificación correcta emprendedor, por lo que estaría cumpliendo la prueba de máxima verosimilitud. La Tabla 5 muestra los resultados prácticos de la utilización de la red. Para cada caso la respuesta pronosticada es «Emprendedor».

1. Las casillas de la diagonal de la clasificación conjunta de los casos son los pronósticos correctos.

2. Las casillas fuera de la diagonal de la clasificación conjunta de los casos son los pronósticos incorrectos.

Tabla 5. Clasificación de los resultados prácticos de la utilización de la red neuronal Table 5. Classification of the Practical Results of Using a Neural Network

\begin{tabular}{|c|c|c|c|c|}
\hline \multirow[b]{2}{*}{ Ejemplo } & \multirow[b]{2}{*}{ Observado } & \multicolumn{3}{|c|}{ Pronosticado } \\
\hline & & No & $\mathrm{Si}$ & $\begin{array}{c}\text { Porcentaje } \\
\text { correcto }\end{array}$ \\
\hline \multirow{3}{*}{ Entrenamiento } & No & 49 & 55 & $47,1 \%$ \\
\hline & $\mathrm{Si}$ & 14 & 179 & $92,7 \%$ \\
\hline & Porcentaje global & $21,2 \%$ & $78,8 \%$ & $76,8 \%$ \\
\hline \multirow{3}{*}{ Pruebas } & No & 19 & 24 & $44,2 \%$ \\
\hline & $\mathrm{Si}$ & 8 & 80 & $90,9 \%$ \\
\hline & Porcentaje global & $20,6 \%$ & $79,4 \%$ & $75,6 \%$ \\
\hline \multirow{3}{*}{ Reserva } & No & 3 & 9 & $25,0 \%$ \\
\hline & $\mathrm{Si}$ & 5 & 33 & $86,8 \%$ \\
\hline & Porcentaje global & $16,0 \%$ & $84,0 \%$ & $72,0 \%$ \\
\hline \multicolumn{5}{|c|}{ Variable dependiente: Emprendedor } \\
\hline
\end{tabular}

Fuente: elaboración propia a partir de la información procesada en Economática.

La importancia de una variable independiente (formación curricular) es una medida que indica cuánto cambia el valor pronosticado por el modelo de la red, para diferentes valores de la variable independiente con la dependiente (emprendedor). En este caso, se observan porcentajes de acierto en clasificación emprendedora por encima del $86 \%$.

En la Figura 2, se muestran los resultados finales de la variable independiente (formación curricular en forma descendente). Los ítems están relacionados con el hecho de que la universidad cuente con convenios para la colaboración con programas que incentivan el emprendimiento, que los alumnos realicen actividades para obtener ingresos extras, practiquen algún deporte, que las instituciones ofrezcan apoyo para la creación de empresas y en menor grado las asignaturas contenidas en el plan de estudios. En contraste, existen estudios que muestran que la educación y formación universitaria no garantizan la vinculación de los estudiantes con el mundo empresarial, dado que gran parte de los graduados prefieren ser empleados en grandes empresas en lugar de intentar crear su propia empresa, originado principalmente por la incertidumbre y riesgos que están asociados a emprender (Abdullah \& Abidin, 2014), y que en general son necesario apoyos e inventivos para que desarrollen un trabajo independiente (Messina y Hochsztain, 2015).

En el aspecto social, se observa en mayor importancia el apoyo familiar y la convivencia en las iniciativas emprendedoras tienen mayor efecto sobre la discriminación entre personas emprendedoras y no emprendedoras. Es consistente con trabajos previos, en donde se muestra un coeficiente positivo y significativo de la tradición familiar que indica cierta ascendencia ligada al 
emprendimiento, lo cual estimula a las futuras generaciones a participar como emprendedores (Martínez Gámez, 2016).

En la dimensión característica emprendedora, solo se obtiene la búsqueda de excelencia en las actividades que realiza como predictora de característica de emprendimiento. Por tanto, la formación universitaria es la que mayor relación tiene en el proceso emprendedor, por lo que se acepta la hipótesis de trabajo.

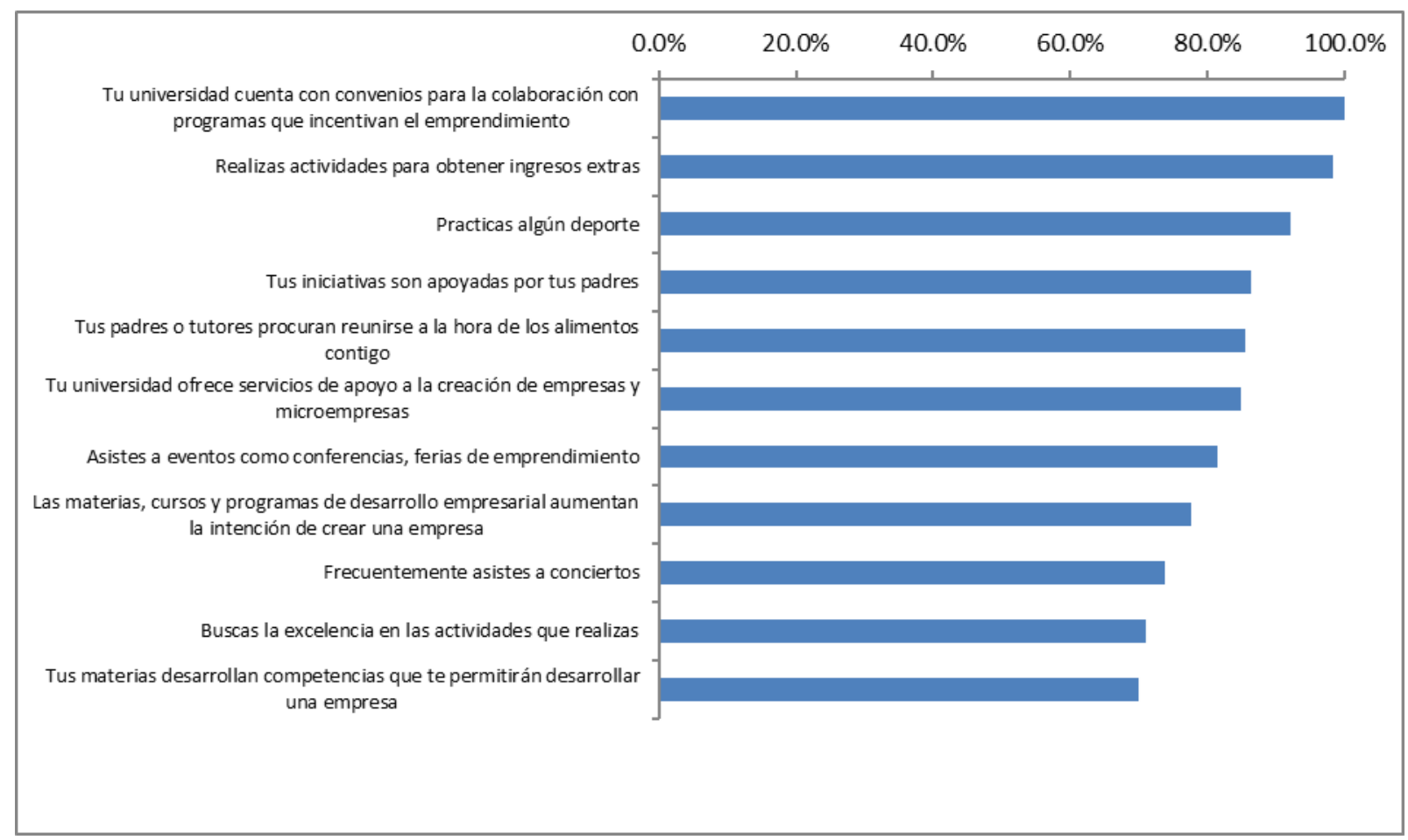

Figura 2. Importancia normalizada

Figure 2. Normalized significance

Fuente: elaboración propia a partir de la información procesada en Economática.

\section{CONCLUSIONES}

Caracterizar la influencia de la formación universitaria en las actitudes emprendedoras a partir de la adquisición de rasgos dinámicos de innovación, propensión al riesgo, personalidad, aspectos psicológicos y demográficos es importante para contribuir a la discusión sobre la importancia de la formación universitaria en el emprendimiento.

Como resultado de la aplicación de las RNA en la clasificación del proceso emprendedor, se valida que la formación universitaria tiene fuerte incidencia en el proceso emprendedor de los estudiantes del programa de Administración en las universidades estudiadas, con un porcentaje de acierto de clasificación del 72 \%, lo que muestra confiabilidad para caracterizar este comportamiento. 
En cuanto al análisis de los resultados de 75 ítems, 11 de ellos permiten reconocer que la formación universitaria que brindan las universidades aporta en gran medida al desarrollo del proceso emprendedor de los jóvenes universitarios, se puede afirmar, en este caso, que la formación práctica que otorga el desarrollarse en las empresas vinculadas, la disciplina de trabajar y obtener recursos económicos adicionales durante sus estudios, ejercitar algún deporte, y las asignaturas contenidas en el plan de estudios son aspectos clave para influir en el emprendimiento y por consecuencia en el desempeño competitivo de los estudiantes. Por ello, las universidades no deben perder de vista la formación integral de los estudiantes y el apoyo a programas que les permitan obtener recursos extraordinarios, como la conformación de incubadoras y la movilidad estudiantil.

De igual forma, es necesario el aspecto social del apoyo familiar para que los jóvenes puedan realizar sin dificultad lo que deseen emprender. Sin embargo, la característica emprendedora de buscar excelencia en lo que realizan tiene un porcentaje menor de influencia. El objetivo planteado se cumple y se comprueba la hipótesis planteada. Sin embargo, la incidencia de estas variables puede cambiar en otras instituciones y en otro tiempo.

A partir del presente estudio, se abren líneas futuras de investigación para analizar el comportamiento estudiante de otras instituciones públicas y privadas nacionales por medio de esta metodología, así como comparar el comportamiento emprendedor en otros países. Se recomienda para futuras investigaciones incrementar el número de variables, y muestra, tanto de universidades como de estudiantes.

\section{REFERENCIAS}

Abdullah, A., \& Abidin, Z. Z. (2014). Factors influencing graduates to start a business: A case of Malaysian public institution of higher learning. En 23rd International Business Information Management Association Conference, IBIMA, España.

Ajzen, I. (1991). The Theory of planned behavior. Organizational Behavior and Human Decision Processes, 50(2), 179-211. https://doi.org/10.1016/0749-5978(91)90020-T

Ayerbe, M., \& Buenetxea, E. (2000). La cultura del trabajo y la actitud emprendedora en el ámbito profesional de la $\quad$ C. A A P. F. V. Recuperado de http://www.gazteaukera.euskadi.eus/contenidos/documentacion/cuaderno sociologico vas co 3/es cu soc3/adjuntos/csv3.pdf

Banco Mundial. (2016). Los emprendedores y las pequeñas empresas impulsan el crecimiento económico $\quad y \quad$ crean empleos. Recuperado de https://www.bancomundial.org/es/news/feature/2016/06/20/entrepreneurs-and-smallbusinesses-spur-economic-growth-and-create-jobs

Benavente, J. M. (2004). El proceso emprendedor en Chile. Recuperado de https://publications.iadb.org/es/publicacion/14735/el-proceso-emprendedor-en-chile 
Buelna, M. \& Ávila, S. (2009). Cultura Emprendedora. Tradición y actualidad. Tiempo y escritura, 8. Recuperado de https://www.azc.uam.mx/publicaciones/tye/tye15/art hist_08.html

Cadavid, L., Díez-Echavarría, L. \& Valencia-Arias, J. A. (2017). Spin-off activities at higher educational institutions: performance implications from a modeling perspective. Journal of Developmental Entrepreneurship, 22(2), 1750013. https://doi.org/10.1142/S1084946717500133

Cantillon, R. (1955). Essai Sur la Nature du Commerce en Général. Economies, sociétés, civilisations, 10(4), 598-600. Recuperado de https://www.persee.fr/doc/ahess 0395$\underline{2649 \quad 1955 \text { num } 10 \quad 4 \quad 2502 \text { t1 } 0598 \quad 0000 \_2}$

Capelleras Segura, J. L., Contín Pilart, I., Martín Sánchez, V. \& Lazarra Kintana, M. (2013). The influence of individual perceptions and the urban/rural environment on nascent entrepreneurship. Journal of Regional Research, (26), 97-113. Recuperado de http://hdl.handle.net/10017/26973

Doh, S. \& Zolnik, E. (2011). Social capital and entrepreneurship: An exploratory analysis. African Journal of Business Management, 5(12), 4961-4975. Recuperado de https://academicjournals.org/journal/AJBM/edition/18 June, 2011

Entrialgo, M. e Iglesias, V. (2016). The moderating role of entrepreneurship education on the antecedents of entrepreneurial intention. International Entrepreneurship and Management Journal, 12(4), 1209-1232. https://doi.org/10.1007/s11365-016-0389-4

Espíritu, R., \& Moreno, H. (2010). La influencia de los rasgos de personalidad, riesgo, control interno y necesidad de logro en la intención emprendedora: un estudio empírico con estudiantes universitarios. México: Congreso Internacional de Contaduría, Administración e Informática. Recuperado de http://congreso.investiga.fca.unam.mx/docs/xv/docs/7.pdf

Estrin, S., Mickiewicz, T. \& Stephan, U. (2016). Human capital in social and commercial entrepreneurship. Journal of Business Venturing, 31(4), 449-467. https://doi.org/10.1016/j.jbusvent.2016.05.003

Fayolle, A. \& Vernier, A. \& Djiane, B. (2004). Les jeunes diplômés de l'enseignement supérieur sontils des créateurs d'entreprise comme les autres? Revue de Gestion 2000, 21(2), 39-56.

Ferry, G., Wieder, R. E., \& Jiménez Silva, M. (1991). El trayecto de la formación. Los enseñantes entre teoría y la práctica. México: Piadós. Gestión 2000.

Fuentes-García, F. J., \& Sánchez-Cañizares, S. M. (2010). Análisis del perfil emprendedor: una perspectiva de género. Estudios de Economía Aplicada, 28(3), 1-27. Recuperado de http://www.redalyc.org/articulo.oa?id=30120334014

García, C., Martínez, A. y Fernández, R. (2007). Análisis de los factores determinantes de la creación de empresas: una evidencia empírica en Castilla y León. In Decisiones basadas en el 
conocimiento y en el papel social de la empresa: XX Congreso anual de AEDEM (p. 40). Asociación Española de Dirección y Economía de la Empresa (AEDEM).

García, J. M. (2018). Teorías y ejercicios prácticos de Dinámica de Sistemas. Recuperado de https://bit.ly/2YATTV2

Giannetti, M. \& Simonov, A. (2004). On the determinants of entrepreneurial activity: Social norms, economic environment and individual characteristics. Swedish Economic Policy Review, 11(2), 269-313. Recuperado de https://ssrn.com/abstract=669503

Gibb, A., \& Ritchie, J. (1982). Understanding the Process of Starting Small Businesses. Small Business Journal, 1(1), 26-45. Recuperado de https://doi.org/10.1177/026624268200100102

Glocker, D. \& Steiner, V. (2007). Self-Employment: Way to End Unemployment? Empirical Evidence from German Pseudo-Panel Data. IZA Discussion Paper No. 2561. Recuperado de https://ssrn.com/abstract=960005

Hamzah, H., Yahya, Z., Sarip, A. G. \& Adnan, Y. M. (2016). Impact of entrepreneurship education programme (EEP) on entrepreneurial intention of real estate graduates. Pacific Rim Property Research Journal, 22(1), 17-29. https://doi.org/10.1080/14445921.2016.1158897

Herman, E. \& Stefanescu, D. (2017). Can higher education stimulate entrepreneurial intentions among engineering and business students? Educational Studies, 43(3), 312-327. https://doi.org/10.1080/03055698.2016.1277134

Hernández-López, D. M., Moncada-Toro, J. F., \& Henao-Colorado, L. C. (2018). Intención emprendedora de los empleados del sector privado de la ciudad de Medellín y su Área Metropolitana. Revista CEA, 4(8), 13-33. https://doi.org/10.22430/24223182.1045

Hornaday, J. A. \& Aboud, J. (1971). Characteristics of successful entrepreneurs. Personnel Psycology, 24(2), 141-153. https://doi.org/10.1111/j.1744-6570.1971.tb02469.x

Lazear, E. (2005). Entrepreneurship. Journal of Labor Economics, 23(4), 649-680. https://doi.org/10.1086/491605

Leal, C. M. y Cepeda, J. M. (2015). Assessing the impact of Information and Communication Technologies on the Portuguese hotel sector: an exploratory analysis with Data Envelopment Analysis. Tourism \& Management Studies, 11(1), 35-43. Recuperado de https://www.tmstudies.net/index.php/ectms/article/view/755/1262

Lederman, D., Messina, J., Pienknagura, S. y Rigolini, J. (2014). El emprendimiento en América Latina. Muchas empresas y poca innovación. Recuperado de https://www.worldbank.org/content/dam/Worldbank/document/LAC/EmprendimientoAme ricaLatina resumen.pdf 
Martínez Gámez, A. (2016). Factores socio-culturales asociados al emprendedor: evidencia empírica para América Latina. Revista Venezolana de Gerencia, 21(74), 312-330. Recuperado de https://produccioncientificaluz.org/index.php/rvg/article/view/21262

McClelland, D. (1961). The Achieving Society. Princeton: D. Van Nostrand Company, Inc.

Mejía Ordoñez, J. P., Arias Marín, C. M., \& Echeverri Sánchez, L. C. (2017). El papel de la educación en creación de empresas en el contexto universitario a partir de los estudios registrados en Scopus. Revista CEA, 3(5), 69-87. https://doi.org/10.22430/24223182.651

Messina, M. \& Hochsztain, E. (2015). Factores de éxito de un emprendimiento: Un estudio exploratorio con base en técnicas de data mining. Tec empresarial, 9(1), 31-40. https://doi.org/10.18845/te.v9i1.2206

Organización para la Cooperación y el Desarrollo Económicos, Banco de Desarrollo de América Latina y Comisión Económica para América Latina y el Caribe. (2016). Perspectivas económicas de América Latina 2017. Juventud, competencias y emprendimiento. Recuperado de https://www.oecd.org/dev/americas/E-book_LEO2017 SP.pdf

Pérez, M., \& Martín, Q. (2003). Aplicaciones de las redes neuronales artificiales a la estadística. Madrid: La Muralla.

Quiceno, H. (1996). Rousseau y el concepto de formación. Revista Educación y Pedagogía, 7, 66-92. Recuperado http://aprendeenlinea.udea.edu.co/revistas/index.php/revistaeyp/article/view/5580

Real Academia Española. (2019). Diccionario. Recuperado de https://dle.rae.es/?id=|F1|1Br

Reboul, O. (1989). Philosophie de I’Educaction. Paris: PUF.

Reyes-Cruz, J. L., Garzón-Castrillón, M. A. \& Tapia-Sánchez, B. (2018). Diseño y validación de una escala tipo Likert para establecer características emprendedoras. Dimensión Empresarial, 16(2), 135-160. https://dx.doi.org/10.15665/dem.v16i2.1599

Ross, B. W. (1995). Entrepreneurship, Management, and the Structure of Payoffs by William J. Baumol. https://doi.org/10.1080/08109029508629199

Santana Vega, L. E., González-Morales, O. \& García, L. F. (2016). Emprendimiento y adolescencia. New approaches in educational research, 5(2), 131-138. https://doi.org/10.7821/naer.2016.7.165

Say, J. B. (1821). A Treatise on Political Economy or the Production, Distribution and Consumption. Philadelphia: New American Edition. 
Scott, M. G. \& Twomey, D. F. (1988). The long-term supply of entrepreneurs: Students' career aspirations in relation to entrepreneurship. Journal of Small Business Management, 26(4), 513. Recuperado de https://search.proquest.com/docview/221002129?accountid=30687

Schumpeter, J. (1934). The Theory of Economic Development. Alemania: Cambridge Mass.

Sudek, R. (2006). Angel Investment Criteria. Journal of Small Business Strategy, 17(2), 89-104. Recuperado de https://libjournals.mtsu.edu/index.php/jsbs/article/view/68

Van Praag, C. M. (1999). Some Classic Views on Entrepreneurship. De Economist, 147(3), 311335. https://doi.org/10.1023/A:1003749128457 